\title{
Could Hyaluronic acid (HA) reduce Bacillus Calmette-Guérin (BCG) local side effects? Results of a pilot study
}

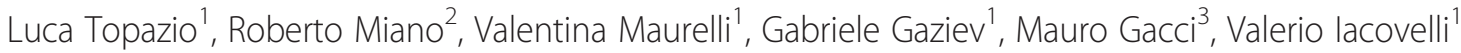 \\ and Enrico Finazzi-Agrò $2,4^{*}$
}

\begin{abstract}
Background: Bacillus Calmette-Guérin (BCG) is considered the most effective treatment to reduce recurrence and progression of non-muscle invasive bladder cancer (NMIBC) but can induce local side effects leading to treatment discontinuation or interruption. Aim of this exploratory study is to investigate if the sequential administration of Hyaluronic acid (HA) may reduce local side effects of BCG.

Methods: 30 consecutive subjects undergoing BCG intravesical administration for high risk NMIBC were randomized to receive BCG only (Group A) or BCG and HA (Group B). A 1 to 10 Visual Analog Scale (VAS) for bladder pain, International Prostate Symptom Score (IPSS) and number of micturitions per day were evaluated in the two groups before and after six weekly BCG instillations. Patients were also evaluated at 3 and 6 months by means of cystostopy and urine cytology.
\end{abstract}

Results: One out of $30(3,3 \%)$ patients in group A dropped out from the protocol, for local side effects. Mean VAS for pain was significantly lower in group B after BCG treatment (4.2 vs. 5.8, $p=0.04)$. Post vs. pre treatment differences in VAS for pain, IPSS and number of daily micturitions were all significantly lower in group B. Three patients in group A and 4 in group B presented with recurrent pathology at 6 month follow up.

Conclusions: These preliminary data suggest a possible role of HA in reducing BCG local side effects and could be used to design larger randomized controlled trials, assessing safety and efficacy of sequential BCG and HA administration.

Trial registration: NCT02207608 (ClinicalTrials.gov) 01/08/2014 Policlinico Tor Vergata Ethics Committee, resolution n 69-2011.

Keywords: BCG, Hyaluronic acid, Non-muscle invasive bladder cancer, Non bacterial cystitis

\section{Background}

Bacillus Calmette-Guérin (BCG) is considered the most effective treatment to increase disease-free interval and reduce progression of non-muscle invasive bladder cancer (NMIBC) [1]. Although considered safe, BCG can produce both local and systemic side effects leading to treatment discontinuation or interruption. The most common local

\footnotetext{
*Correspondence: efinazzi@tin.it

${ }^{2}$ Department of Experimental Medicine and Surgery, Tor Vergata University, Rome, Italy

${ }^{4}$ Unit for Functional Urology, Department of Urology, Policlinico Tor Vergata, Rome, Italy

Full list of author information is available at the end of the article
}

side-effects of BCG intravesical instillations include cystitis, characterized by irritative voiding symptoms and hematuria, which occur in approximately $75 \%$ of all patients. More rarely, serious local adverse events as a result of BCG infection, such as symptomatic granulomatous prostatitis and epididymo-orchitis, might occur and require permanent discontinuation of BCG treatment. Systemic side-effects include flu-like symptoms, such as general malaise and fever, occuring in approximately $40 \%$ of patients. A high persistent fever might be related to BCG infection or sepsis. Local and systemic side-effects might lead to discontinue intravesical BCG treatment in approximately $20 \%$ of patients [2]. Up to $54 \%$ of the

\section{Biomed Central}

(c) 2014 Topazio et al.; licensee BioMed Central Ltd. This is an Open Access article distributed under the terms of the Creative Commons Attribution License (http://creativecommons.org/licenses/by/2.0), which permits unrestricted use, distribution, and reproduction in any medium, provided the original work is properly credited. The Creative Commons Public Domain Dedication waiver (http://creativecommons.org/publicdomain/zero/1.0/) applies to the data made available in this article, unless otherwise stated. 
patients undergoing intravesical therapy with chemotherapeutic agents to treat superficial bladder tumours can be affected by nonbacterial cystitis [3].

Several solutions have been proposed to reduce the occurrence of side effects from BCG with the aim to limit BCG discontinuation and the concomitant discomfort during endovesical treatment. Some Authors have proposed to avoid BCG administration in case of TUR within previous 2 weeks, traumatic catheterization, macroscopic hematuria, urethral stenosis, active tuberculosis, prior Bacillus Calmette-Guérin sepsis, immuno-suppression or urinary tract infection [4]. Other procedures include the prophylactic administration of isoniazid [5] or ofloxacin $[6,7]$ or usually involve BCG dose reductions [8]. In common practice antimicrobials, anticholinergics, anaesthetics and analgesics are often used to relieve patients' symptoms.

Glycosaminoglycan (GAG) substitution therapy is an emerging treatment of Bladder Pain Syndrome/Interstitial Cystitis (BPS/IC) and response rates between 30\% and $80 \%$ have been described with intravesical administration of various GAGs (hyaluronic acid, pentosan polysulfate, heparin, chondroitin sulfate, and dimethyl sulfoxide) $[9,10]$. Few papers report the results of GAG substitution therapy in the treatment of radiation and chemical cystitis $[9,10]$. To our knowledge, to date, only two papers have described GAG use in the treatment of BCG local side effects; this papers show very good results, with significant reduction of lower urinary tract symptoms after intravesical administration of HA [11,12].

Aim of the present randomized pilot study was to evaluate if the sequential administration of HA and BCG could be safe in prevention of early recurrence and progression of bladder tumor, and safe in reduction of local side-effects in patients with high risk NMIBC.

\section{Methods}

This is a prospective randomized pilot study approved by our institutional review board (Policlinico Tor Vergata Ethics Committee, resolution $n^{\circ}$ 69-2011). Patients with a diagnosis of a intermediate/high risk NMIBC, according to the European Organization for Research and Treatment of Cancer (EORTC) score [13] after transurethral resection of bladder tumor (TURBT) were eligible for this study.

Inclusion criteria were:

1. Hystologically proven non-muscle invasive bladder cancer;

2. Indication to intravesical instillation of BCG according to EAU guidelines;

3. Age $>18$ years;

4. Willingness, to participate to the study;

5. Written informed consent.
Exclusion criteria were:

1. Previous or ongoing BCG or different intravesical instillations;

2. Urinary tract infections (UTI) or other known pathologies of the lower urinary tract;

3. Indication for a radical cystectomy;

4. Severe systemic disorders, including neurological pathologies, kidney, liver or heart failure;

5. Contraindications to BCG use.

Patients enrolled in the study started the intravesical therapies within 4 weeks from the TURBT and were randomized in two groups. We a priori decided to stop this pilot study after reaching the number of 15 patients in each group. Group A was sent to receive BCG (Immucist $^{\circ} 81 \mathrm{mg}$, Sanofi-Aventis Group) alone and Group B to receive BCG (see previous reference) and HA $40 \mathrm{mg}$ (Cystistat, Mylan, Pittsburgh, PA, U.S.A.). BCG strain used in our study was Connaught $3,4 \pm 3 \times 10$ (8) CFU, $81 \mathrm{mg}$ $\left(\right.$ Immucist $\left.t^{\circ}\right)$ in $50 \mathrm{cc}$ of saline. BCG was administered by means of a 2 ways 14 Ch Foley catheter latex and left inside the bladder for 60 minutes. After 60 minutes BCG was evacuated, the bladder washed with saline and the catheter removed. HA was administered intravesically after every BCG evacuation in Group B. After HA administration and catheter removal, patients were instructed to maintain HA in the bladder as long as possible, for at least 2 hours after administration. In Group A normal saline was administered, so that all patients were blinded to the administration of HA. Both groups received an induction course of 6 weekly instillations of BCG.

A 1 to 10 Visual Analog Scale (VAS) for bladder pain was considered the primary outcome measure of the study and was evaluated in both groups before and after the treatment. International Prostate Symptom Score (IPSS) and number of micturitions per day were also evaluated in both groups before starting and at the end of the induction cycle of BCG instillations.

Patients were evaluated at three and six months by means of cystoscopy and urine cytology for oncologic follow-up. The number of patients with bladder tumor recurrence was recorded in both groups at 6 month follow-up.

Data are presented as mean \pm standard deviation (SD). The statistical analysis was conducted with a t Student Test using a software Stata 12.0, and considering as significant a $p$ value $\leq 0.05$.

Our manuscript adheres to CONSORT guidelines. CONSORT checklist is shown in Additional file 1.

\section{Results and discussion}

30 subjects were enrolled in the study between January and December 2011. Mean age was 67 (range 54-81): 23 
(76\%) were male while 7 (24\%) female. All Patients had $\mathrm{BCa}$ with intermediate/high risk of progression according to the European Organization for Research and Treatment of Cancer (EORTC) score. Fifteen patients (12 male and 3 female) were randomly assigned to Group $\mathrm{A}$ and 15 patients (11 male and 4 female) to Group B. Clinical and pathologic features of patients are shown in Table 1.

Only one out of $30(3,3 \%)$ patients in Group A dropped out from the protocol, for local side effects (urgency, frequency and burning micturition), while all Patients in Group B completed the induction cycle with intravesical BCG. The results are shown in Table 2.

Mean pre-treatment VAS was comparable in the two groups $(4.46(2 ; 10)$ for Group A and $4.86(2 ; 8)$ for Group $\mathrm{B}, \mathrm{p}=0.56)$. After treatments mean VAS was significantly lower in group B $(4.2(2 ; 7)$ vs. $5.53(4 ; 7)$ in Group $\mathrm{A}, \mathrm{p}=0.04)$. VAS post treatment was increased in Group $A$ and reduced in Group B: difference in post vs pretreatment VAS was significantly lower in Group B $(-0.66(-4 ; 1)$ vs. $1.06(-4 ; 3)$ in Group A, p = 0.0001).
Mean pre-treatment IPSS was comparable in both groups $(13.93(8 ; 24)$ in Group A and $14.73(9 ; 22)$ in Group $B, p=0.6)$. After treatment, mean IPSS was slightly lower in group B $(15.26(11 ; 21)$ vs. $17.46(13 ; 21)$ in Group A, even if the difference was not statistically significant $p=0.1$ ). IPSS increase post treatment was significantly lower in Group B than in Group A $(0.53$ $(-3 ; 2)$ vs. $3.53(-3 ; 10)$ in Group A, p=0.02).

Mean pre-treatment number of daily micturitions was comparable in the two groups $(10.26(7 ; 15)$ for Group A and $10.8(7 ; 16)$ for Group B, p = 0.53). After treatment, we failed to find a significant difference in number of daily micturitions between the two groups $(11.26(9 ; 13)$ in Group A and $10.73(7 ; 14)$ in Group B, p=0.44). On the other hand, there was no increase in number of daily micturitions in Group B and a slight increase in Group A: this difference was significant $(-0.066(-2 ; 1)$ for Group B and $1(-2 ; 5)$ for Group A, p = 0.04).

At 6 month follow-up 3/15 (21\%) pts of Group A (including the patient who dropped out from the induction

Table 1 Clinical and pathologic features of pts

\begin{tabular}{|c|c|c|c|}
\hline & Tot & Group A (BCG) & Group B $(B C G+H A)$ \\
\hline \multicolumn{4}{|l|}{ Gender } \\
\hline $\bar{M}$ & $23(76 \%)$ & $12(80 \%)$ & $11(73 \%)$ \\
\hline$F$ & $7(24 \%)$ & $3(20 \%)$ & $4(27 \%)$ \\
\hline \multicolumn{4}{|l|}{ Age } \\
\hline Mean & 67 & 66 & 68 \\
\hline Range & $54-81$ & $54-75$ & $60-81$ \\
\hline \multicolumn{4}{|l|}{$N^{\circ}$ of tumors } \\
\hline Single & $9(30 \%)$ & $5(33 \%)$ & $4(27 \%)$ \\
\hline Multiple & $21(70 \%)$ & $10(67 \%)$ & $11(53 \%)$ \\
\hline \multicolumn{4}{|l|}{ Tumor diameter } \\
\hline Mean & $1,5 \mathrm{~cm}$ & $1,5 \mathrm{~cm}$ & $1,5 \mathrm{~cm}$ \\
\hline Range & $5 \mathrm{~cm}-50 \mathrm{~cm}$ & $5 \mathrm{~cm}-40 \mathrm{~cm}$ & $5 \mathrm{~cm}-50 \mathrm{~cm}$ \\
\hline \multicolumn{4}{|l|}{$\overline{p T}$} \\
\hline $\mathrm{Ta}$ & $17(56 \%)$ & $9(60 \%)$ & $8(53 \%)$ \\
\hline$T 1$ & $13(44 \%)$ & $6(40 \%)$ & $7(47 \%)$ \\
\hline CIS & $8(27 \%)$ & $3(20 \%)$ & $5(33 \%)$ \\
\hline \multicolumn{4}{|l|}{ WHO grade 1973} \\
\hline G1 & $8(27 \%)$ & $4(27 \%)$ & $4(27 \%)$ \\
\hline G2 & $10(33 \%)$ & $6(40 \%)$ & $4(27 \%)$ \\
\hline G3 & $12(40 \%)$ & $5(33 \%)$ & $7(46 \%)$ \\
\hline \multicolumn{4}{|c|}{ EORTC recurrence score } \\
\hline$\leq 9$ intermediate & $10(33 \%)$ & $6(40 \%)$ & $4(27 \%)$ \\
\hline 10-17 high & $20(67 \%)$ & $9(60 \%)$ & $11(73 \%)$ \\
\hline \multicolumn{4}{|c|}{ EORTC progressive score } \\
\hline$\leq 6$ intermediate & $11(36 \%)$ & $6(40 \%)$ & $5(33 \%)$ \\
\hline 7-23 high & 19 (64\%) & $9(60 \%)$ & 10 (67\%) \\
\hline
\end{tabular}


Table 2 Results of VAS, IPSS and bladder diaries at baseline and at the end of BCG induction cycle

\begin{tabular}{|c|c|c|c|c|c|c|c|c|c|}
\hline \multirow[b]{2}{*}{ Mean (SD) } & \multirow{2}{*}{$\begin{array}{c}\text { Group A } \\
\text { Pre }\end{array}$} & \multirow{2}{*}{$\begin{array}{c}\text { Group B } \\
\text { Pre }\end{array}$} & \multirow[t]{2}{*}{$p$} & \multirow{2}{*}{$\begin{array}{c}\text { Group A } \\
\text { Post }\end{array}$} & \multirow{2}{*}{$\begin{array}{c}\text { Group B } \\
\text { Post }\end{array}$} & $p$ & Group A & Group B & \multirow[t]{2}{*}{$p$} \\
\hline & & & & & & \multicolumn{3}{|c|}{ Difference } & \\
\hline $\operatorname{VAS}(1-10)$ & $4.5( \pm 2)$ & $4.9( \pm 1.8)$ & .56 & $5.8( \pm 1)$ & $4.2( \pm 1.6)$ & .04 & $1.5( \pm 0.7)$ & $-0.7( \pm 1.6)$ & .0001 \\
\hline IPSS & $13.9( \pm 4.4)$ & $14.7( \pm 4.0)$ & .60 & $17.5( \pm 2.6)$ & $15.3( \pm 4)$ & .10 & $3( \pm 3.5)$ & $0.53( \pm 1.6)$ & .02 \\
\hline$n^{\circ}$ daily micturitions & $10.3( \pm 2.2)$ & $10.8( \pm 2.4)$ & .53 & $11.5( \pm 1.3)$ & $10.9( \pm 2.1)$ & .44 & $1.23( \pm 1.7)$ & $0.13( \pm 1)$ & .04 \\
\hline
\end{tabular}

BCG cycle for severe LUTS) and 4/15 (26.7\%) pts in Group B had a recurrence of BCa. None of them had $\mathrm{BCa}$ progression to muscle-invasive disease.

Several trials in the last two decades have investigated BCG efficacy in reducing the risk of recurrence and progression in intermediate/high risk $\mathrm{BCa}$ showing that BCG after TUR is superior to TUR alone or TUR and conventional chemotherapy $[14,15]$. Two meta-analyses have demonstrated that BCG therapy prevents, or at least delays, the risk of tumour progression $[16,17]$.

Despite of its great efficacy, BCG therapy has potential local and systemic side effects that may either lead to treatment cessation in up to $30 \%$ of patients or lead to a delay or reduction in the number of instillations in $55-83 \%$ of patients [18].

The risk of increased toxicity during maintenance has been questioned. According to the results of a European Organization for Research and Treatment of Cancer (EORTC) phase 3 trial [18], local side effects of BCG do not increase during maintenance and systemic side effects are more frequent during the first 6 mo of treatment. However, a significant proportion of patients $(84 \%$ [19], 67.3\% [18]), 86\% [20] in the three most representative series) failed to complete the 3-yr maintenance course for various reasons.

Several options have been proposed to decrease the occurrence of BCG side effects including the prophylactic administration of isoniazid or ofloxacin, modifications to the BCG treatment schedule and dose reductions. The concomitant administration of isoniazid, has not been found to decrease the incidence of BCG related sideeffects [5]. In contrast, ofloxacin reduced the incidence of moderate to severe BCG related adverse events when given prophylactically in a randomized double blind trial in 115 patients [6,7]. However, further studies are required to confirm these initial findings and to ensure that there is no impairment of treatment efficacy. Martinez-Pineiro et al. investigated the BCG side effects rate using a reduced dose of BCG. They found significantly less toxicity on the reduced dose but recommended the use of a full dose in the treatment of high-risk patients [8]. In a second study in high-risk patients with T1G3 tumors and/or carcinoma in situ (CIS) they concluded that one-third dose was indeed as effective as full dose, but was associated with significantly less toxicity [21]. The final results of an EORTC-GU Cancers
Group Randomized Study of Maintenance Bacillus Calmette-Guerin in Intermediate- and High-risk Ta, T1 Papillary Carcinoma of the Urinary Bladder showed no significant differences in toxicity between $1 / 3$ dose and full dose (FD) BCG. $1 / 3$ dose with $1 \mathrm{yr}$ of maintenance was considered suboptimal compared with the standard FD during 3 yr [22].

Despite of this great number of papers on BCG toxicity in literature, only two papers investigated the possible use of HA for treatment of BCG induced cystitis $[11,12]$ showing interesting results.

GAGs have been used extensively in BPS/IC with response rates between $30 \%$ and $80 \%$ described with intravesical administration of various substances such as HA, PPS, heparin; chondroitin sulfate, and DMSO [23,24]. The efficacy of HA is based on several mechanisms that aim on the urothelial function disorder present in BPS/ IC: on one side, HA reinforces the urine-tissue barrier by integration in the GAG layer on the luminal surface and the base of urothelial cells; on the other side, unique anti-inflammatory mechanisms have been identified, like inhibition of leukocyte migration, adherence of immune complexes, and binding to specific receptors (I-CAM 1, CD 44) involved in the inflammatory process [25-27].

Moreover studies on rat models have shown that HA can inhibit bladder mast cell activation as well as the inflammatory mediator release of urinary histamine, rat mast cell protease-I and IL-6 [28] thus reducing their pro-inflammatory activity.

Because of GAGs documented anti-inflammatory and protective activity on the urothelium we considered the possible use of these devices on the treatment of BCG induced cystitis, even if one can argue that reducing BCG side-effects can even impair BCG efficacy.

A correlation between BCG side-effects and treatment efficacy has been reported by various authors, suggesting that local side-effects have a significantly longer time to first recurrence. However, patients with a better outcome remain on treatment for a longer period of time and receive more $\mathrm{BCG}$, thus increasing their risk of developing side-effects. Neither local nor systemic BCG toxicity before 6 months was found to be a prognostic factor for subsequent recurrence. Thus, it is not possible to confirm that BCG toxicity is actually responsible for an improved outcome and a causal effect cannot be inferred from the data [29]. 
Our preliminary results show a possible role of HA in reducing $\mathrm{BCG}$ side-effects during the induction cycle when their frequency is higher than in the maintenance period [18]. As described in the Results and discussion section, VAS for bladder pain was significantly lower, at the end of the induction cycle, in Group B (patients treated with HA). It is important to underline that baseline VAS was relatively high because obtained in patients who recently underwent a TURBT. Interestingly, patients in Group B showed a reduction, whilst those in Group A presented an increase of VAS for pain. Indeed we failed to show between groups a statistically significant difference in IPSS and number of daily micturitions; nevertheless, the use of HA seems to have reduced the appearance of lower urinary tract symptoms in Group B, with an increase of IPSS of only 0.53 points vs. a significantly higher increase in group A (3.53). The same could be said about number of daily micturitions that remained almost unchanged in Group B whilst was increased after treatment in Group A. These results could indicate a protective function of HA on the urothelium of the bladder against the irritative activity of BCG.

One can argue that even ofloxacin when given prophylactically before each BCG administration [6,7] can achieve good results with a lower cost for the Health Care System. Actually the use of Ofloxacin has significantly decreased the incidence of class 2 or higher AEs, but has not improved class 1 AEs [6] while HA in our paper has demonstrated to significantly reduce class 1 AEs. Moreover it should be discussed if the prophylactic use of Ofloxacin for 9 weeks could increase bacterial resistance.

These very preliminary data seem to support a possible role of HA in reducing BCG local side effects. Nevertheless, our study has some limitations:

1. We have only a short follow up time;

2. It is a pilot study, with no power calculation;

3. Does not provide data on maintenance treatment;

4. Does not provide data on possible long term interactions of HA with BCG efficacy;

5. It remains to be seen whether the administration of HA after BCG is worth the benefit and increased costs.

The only outcome measures evaluated (VAS, IPSS, daily micturitions) may have not detected important efficacy end points, such as quality of life and relief of painful micturition.

For all these reasons, no definitive conclusions should be drawn. Nevertheless the data coming from this study should be used to design a randomized controlled study aimed to verify this hypothesis.

\section{Conclusions}

This pilot study provides, for the first time to our knowledge, evidence of a possible reduction of BCG local adverse events by using a sequential administration of HA. Larger randomized controlled study, designed on the basis of this pilot study are needed to assess how clinically significant the association of HA to BCG could be. In other words, further studies will have to investigate if this association is worthy, having proven (statistically and clinically) significant reduction of local adverse events of BCG, possibly causing a reduction of the number of patients who have to suspend or discontinue the treatment with BCG, with a consequent reduction of its efficacy. Furthermore, a possible interaction of HA with BCG efficacy must be excluded before this association could become part of a standard care. In conclusion, this pilot study is only the first step, but further research is needed to investigate the exact potentialities and role of $\mathrm{HA}$ administered in patients treated by means of BCG for NMIBC.

\section{Additional file}

Additional file 1: A complete CONSORT Checklist.

\section{Abbreviations}

HA: Hyaluronic acid; BCG: Bacillus Calmette-Guérin; NMIBC: Non-muscle invasive bladder cancer; VAS: Visual analog scale; IPSS: International Prostate Symptom Score; GAG: Glycosaminoglycan; BPS/IC: Bladder pain syndrome/ interstitial cystitis; EORTC: European Organization for Research and Treatment of Cancer; TUR: Trans-urethral resection; BCa: Bladder cancer.

\section{Competing interests}

Enrico Finazzi-Agrò is consultant for Mylan Inc. Luca Topazio, Roberto Miano, Valentina Maurelli, Gabriele Gaziev, Valerio lacovelli and Mauro Gacci declare that they have no conflict of interest.

\section{Authors' contributions}

LT has been involved in drafting the manuscript. VI and GG have made substantial contributions to acquisition and analysis of data. VM has made substantial contributions to conception and design of the study. EFA, MG and RM have made substantial contributions to conception and design and interpretation of data; they have been involved in revising the manuscript critically for important intellectual content. All authors read and approved the final manuscript.

\section{Funding}

Departmental; Cystistat supplied by Mylan, Pittsburgh, PA, U.S.A.

\section{Author details}

${ }^{1}$ School of Specialization in Urology, Tor Vergata University, Rome, Italy. ${ }^{2}$ Department of Experimental Medicine and Surgery, Tor Vergata University, Rome, Italy. ${ }^{3}$ Department of Urology, University of Florence, Florence, Italy. ${ }^{4}$ Unit for Functional Urology, Department of Urology, Policlinico Tor Vergata, Rome, Italy.

Received: 24 January 2014 Accepted: 7 August 2014

Published: 13 August 2014

\section{References}

1. Gontero P, Bohle A, Malmstrom PU, O'Donnell MA, Oderda M, Sylvester R, Witjes F: The role of bacillus Calmette-Guérin in the treatment of non-muscle-invasive bladder cancer. Eur Urol 2010, 57(3):410-429. doi:10.1016/j.eururo.2009.11.023. Epub 2009 Nov 13.

2. Lamm DL, van der Meijden PM, Morales A, Brosman SA, Catalona WJ, Herr HW, Soloway MS, Steg A, Debruyne FM: Incidence and treatment 
of complications of bacillus Calmette-Guerin intravesical therapy in superficial bladder cancer. J Urol 1992, 147(3):596-600.

3. Drake MJ, Nixon PM, Crew JP: Drug-induced bladder and urinary disorders. Incidence, prevention and management. Drug Saf 1998, 19(1):45-55.

4. Witjes JA, Palou J, Soloway M, Lamm D, Brausi M, Spermon JR, Persad R, Buckley R, Akaza H, Colombel M, Böhle A: Clinical practice recommendations for the prevention and management of intravesical therapy-associated adverse events. Eur Urol Supp/ 2008, 7(10):667-674.

5. Vegt PD, van der Meijden AP, Sylvester R, Brausi M, Höltl W, de Balincourt C: Does isoniazid reduce side effects of intravesical bacillus Calmette-Guerin therapy in superficial bladder cancer? Interim results of European Organization for Research and Treatment of Cancer Protocol 30911. J Urol 1997, 157(4):1246-1249.

6. Colombel M, Saint F, Chopin D, Malavaud B, Nicolas L, Rischmann P: The effect of ofloxacin on bacillus Calmette-Guérin induced toxicity in patients with superficial bladder cancer: results of a randomized, prospective, double blind, placebo controlled, multicenter study. J Urol 2006, 176:935.

7. O'Donnell M: Does ofloxacin protect against BCG related toxic effects in patients with bladder cancer? Nat Clin Pract Urol 2007, 4:304.

8. Martínez-Piñeiro JA, Flores N, Isorna S, Solsona E, Sebastián JL, Pertusa C, Rioja LA, Martínez-Piñeiro L, Vela R, Camacho JE, Nogueira JL, Pereira I, Resel L, Muntañola P, Galvis F, Chesa N, De Torres JA, Carballido J, Bernuy C, Arribas S, Madero R, for CUETO (Club Urológico Español de Tratamiento Oncológico): Long-term follow-up of a randomized prospective trial comparing a standard $81 \mathrm{mg}$ dose of intravesical bacille CalmetteGuérin with a reduced dose of $27 \mathrm{mg}$ in superficial bladder cancer. BJU Int 2002, 89(7):671-680

9. lacovelli V, Topazio L, Gaziev G, Bove P, Vespasiani G, Finazzi Agrò E: Intravesical glycosaminoglycans in the management of chronic cystitis. Minerva Urol Nefrol 2013, 65(4):249-262.

10. Madersbacher H, van Ophoven A, van Kerrebroeck PE: GAG layer replenishment therapy for chronic forms of cystitis with intravesical glycosaminoglycans-a review. Neurourol Urodyn 2013, 32(1):9-18. doi:10.1002/nau.22256. Epub 2012 Jul 10.

11. Sommariva ML, Sandri SD, Guerrer CS: Treatment of acute iatrogenic cystitis secondary to bladder chemo-immuno-instillation or pelvic radiotherapy. Urologia 2010, 77:187.

12. Fowler $\mathrm{S}$, Daukeh $\mathrm{M}$, Thompson $\mathrm{A}$ : Intravesical sodium hyaluronate (Cystistat ${ }^{\oplus}$ ) as a treatment for chronic BCG Cystitis: a discussion of two successful cases. [http://www.bjui.org/ContentFullltem.aspx?id=638]

13. Sylvester RJ, van der Meijden AP, Oosterlinck W, Witjes JA, Bouffioux C, Denis L, Newling DW, Kurth K: Predicting recurrence and progression in individual patients with stage Ta T1 bladder cancer using EORTC risk tables: a combined analysis of 2596 patients from seven EORTC trials. Eur Urol 2006, 49(3):466-4755. discussion 475-7. Epub 2006 Jan 17.

14. Shelley MD, Kynaston H, Court J, Wilt TJ, Coles B, Burgon K, Mason MD: A systematic review of intravesical bacillus Calmette-Guérin plus transurethral resection vs transurethral resection alone in Ta and T1 bladder cancer. BJU Int 2001, 88(3):209-216.

15. Han RF, Pan JG: Can intravesical bacillus Calmette-Guérin reduce recurrence in patients with superficial bladder cancer? A meta-analysis of randomized trials. Urology 2006, 67(6):1216.

16. Böhle A, Bock PR: Intravesical bacillus Calmette-Guerin versus mitomycin $C$ in superficial bladder cancer: formal meta-analysis of comparative studies on tumour progression. Urology 2004, 63(4):682.

17. Sylvester RJ, van der Meijden AP, Lamm DL: Intravesical bacillus Calmette-Guerin reduces the risk of progression in patients with superficial bladder cancer: a meta-analysis of the published results of randomized clinical trials. J Urol 2002, 168(5):1964.

18. Van der Meijden APM, Sylvester RJ, Oosterlinck W, Hoeltl W, Bono AV, EORTC Genito-Urinary Tract Cancer Group: Maintenance bacillus Calmette-Guerin for TaT1 bladder tumors is not associated with increased toxicity: results from a European Organisation for Research and Treatment of Cancer Genito-Urinary Group phase III trial. Eur Urol 2003, 44:429.

19. Lamm DL, Blumenstein BA, Crissman JD, Montie JE, Gottesman JE, Lowe BA, Sarosdy MF, Bohl RD, Grossman HB, Beck TM, Leimert JT, Crawford ED Maintenance bacillus Calmette-Guerin immunotherapy for recurrent TA, T1 and carcinoma in situ transitional cell carcinoma of the bladder: a randomized Southwest Oncology Group Study. J Urol 2000, 163(4):1124-1129.
20. Saint F, Irani J, Patard JJ, Salomon L, Hoznek A, Zammattio S, Debois H, Abbou CC, Chopin DK: Tolerability of bacille Calmette-Guérin maintenance therapy for superficial bladder cancer. Urology 2001, 57(5):883-888.

21. Martínez-Piñeiro JA, Martínez-Piñeiro L, Solsona E, Rodríguez RH, Gómez JM, Martín MG, Molina JR, Collado AG, Flores N, Isorna S, Pertusa C, Rabadán M, Astobieta A, Camacho JE, Arribas S, Madero R, Club Urológico Español de Tratamiento Oncológico (CUETO): Has a 3-fold decreased dose of bacillus Calmette-Guerin the same efficacy against recurrences and progression of T1G3 and Tis bladder tumors than the standard dose? Results of a prospective randomized trial. J Urol 2005, 174(4 Pt 1):1242-1247.

22. Oddens J, Brausi M, Sylvester R, Bono A, van de Beek C, van Andel G, Gontero P, Hoelt W, Turkeri L, Marreaud S, Collette S, Oosterlinck W: Final results of an EORTC-GU cancers group randomized study of maintenance bacillus Calmette-Guérin in intermediate- and high-risk $\mathrm{Ta}, \mathrm{T} 1$ papillary carcinoma of the urinary bladder: one-third dose versus full dose and 1 year versus 3 years of maintenance. Eur Urol 2013, 63(3):462-472. doi: 10.1016/j.eururo.2012.10.039. Epub 2012 Nov 2.

23. Toft BR, Nordling J: Recent developments of intravesical therapy of painful bladder syndrome/interstitial cystitis: a review. Curr Opin Urol 2006, 16:268.

24. Nickel JC, Egerdie B, Downey J, Singh R, Skehan A, Carr L, Irvine-Bird K: A real-life multicentre clinical practice study to evaluate the efficacy and safety of intravesical chondroitin sulphate for the treatment of interstitial cystitis. BJU Int 2009, 103(1):56-60. doi:10.1111/j.1464-410X.2008.08028.x. Epub 2008 Sep 3.

25. Hurst RE: Structure, function, and pathology of proteoglycans and glycosaminoglycans in the urinary tract. World J Urol 1994, 12:3.

26. Leppilahti M, Hellström P, Tammela TU: Effect of diagnostic hydrodistension and four intravesical Hyaluronan Instillations on bladder ICAM-1 intensity and association of ICAM-1 intensity with clinical response in patients with interstitial cystitis. Urology 2002, 60:46.

27. Schulz A, Vestweber AM, Dressler D: Anti-inflammatory action of a hyaluronic acid-chondroitin sulphate preparation in an in vitro bladder model. Akt Urol 2009, 40(2):109.

28. Boucher WS, Letourneau R, Huang M, Kempuraj D, Green M, Sant GR, Theoharides TC: Intravesical sodium hyaluronate inhibits the rat urinary mast cell mediator increase triggered by acute immobilization stress. J Urol 2002, 167(1):380-384.

29. Sylvester RJ, van der Meijden APM, Oosterlinck W, Hoelt W, Bono AV, EORTC Genito-Urinary Tract Cancer Group: The side effects of bacillus Calmette-Guérin in the treatment of TaT1 bladder cancer do not predict its efficacy: results from a European Organization for Research and Treatment of Cancer Genito-Urinary Group phase III trial. Eur Urol 2003, 44:423.

doi:10.1186/1471-2490-14-64

Cite this article as: Topazio et al:: Could Hyaluronic acid (HA) reduce Bacillus Calmette-Guérin (BCG) local side effects? Results of a pilot study. BMC Urology 2014 14:64

\section{Submit your next manuscript to BioMed Central and take full advantage of:}

- Convenient online submission

- Thorough peer review

- No space constraints or color figure charges

- Immediate publication on acceptance

- Inclusion in PubMed, CAS, Scopus and Google Scholar

- Research which is freely available for redistribution 